\title{
'Hypothesis Article:Therapeutic effect of POL101 eyedrops, anti-VEGF agents, in vitro and in vivo models'
}

\author{
DONGIN LEE ${ }^{1}$, Ji-Yeon Yu ${ }^{1}$ Yu-sun Kang ${ }^{1}$, Hee Kyung Park ${ }^{2}$, and Hye Joo Kim \\ ${ }^{1}$ Seasun Therapeutics Inc \\ ${ }^{2}$ SEASUN BIOMATERIALS, INC
}

August 17, 2020

\begin{abstract}
Background and Purpose: Choroidal neovascularization (CNV) is the leading cause of blindness in patients with age-related macular degeneration (AMD). Several studies reported increased levels of vascular endothelial growth factor (VEGF) in retinal pigment epithelium (RPE) from AMD patients. The aim of this study was to investigate the efficacy of the VEGF-A targeted peptide nucleic acid (PNA) drug, POL101 eyedrops, in retinal pigment epithelial cell and laser-induced CNV animal model. Experimental Approach: POL101 is a PNA based-Antisense oligonucleotide (ASO) drug which chemically improved properties for stability and delivery to tissue through nano-grade particle size. To evaluate the efficacy of POL101, we performed to in vitro assay in retinal pigment cell and Fundus fluorescein angiography (FFA), optical coherence tomography (OCT) scanning, and electroretinography (ERG) in CNV-induced lesion. Key Results: First, the efficacy of POL101 was verified in ARPE-19 and HUVEC cells. And then, we confirmed that POL101 eyedrops was significantly decreased CNV area to $54.2 \%$ compared with vehicle in laser-induced CNV lesion and reduced VEGF level in choroid tissue without cellular apoptosis. Also, Photocoagulation demonstrated that the average CNV lesion area was reduced and recovered retina function in POL101 group. The mRNA expression of VEGF in mouse retina, intracellular adhesion molecule-1 (ICAM-1), matrix metallopeptidases -2 (MMP-2) and 9 (MMP-9) were significantly reduced in POL101 eyedrops-treated group. Conclusion and Implications: In conclusion, POL101 eyedrops was effectively suppressed VEGF-mediated CNV than aflibercept and has the potential to be effective without side effects and could replace biologics.
\end{abstract}

\section{Hosted file}

manuscript_200814.pdf available at https://authorea.com/users/351318/articles/475983-hypothesis-article-therapeutic-effect-of-pol101-eyedrops-anti-vegf-agents-in-vitro-andin-vivo-models 
(a)

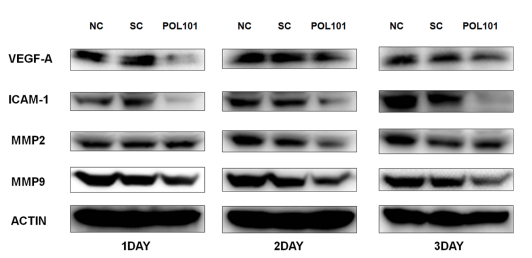

(c)

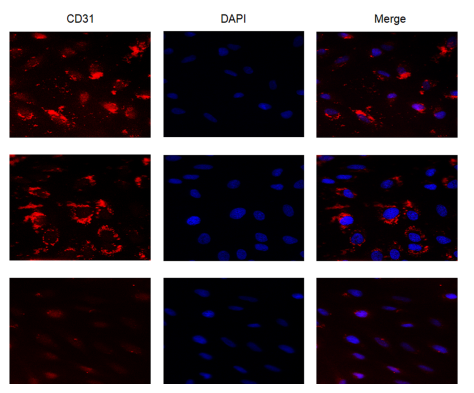

(b)

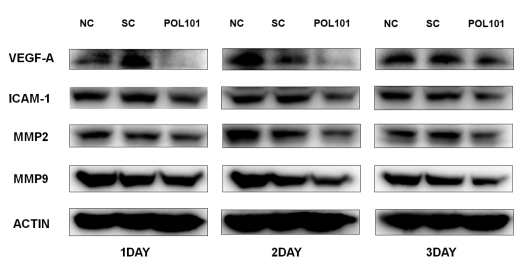

(d)

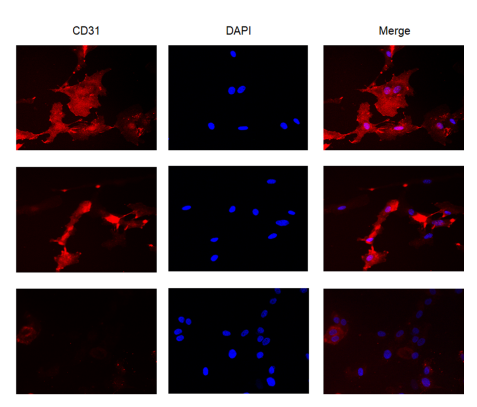



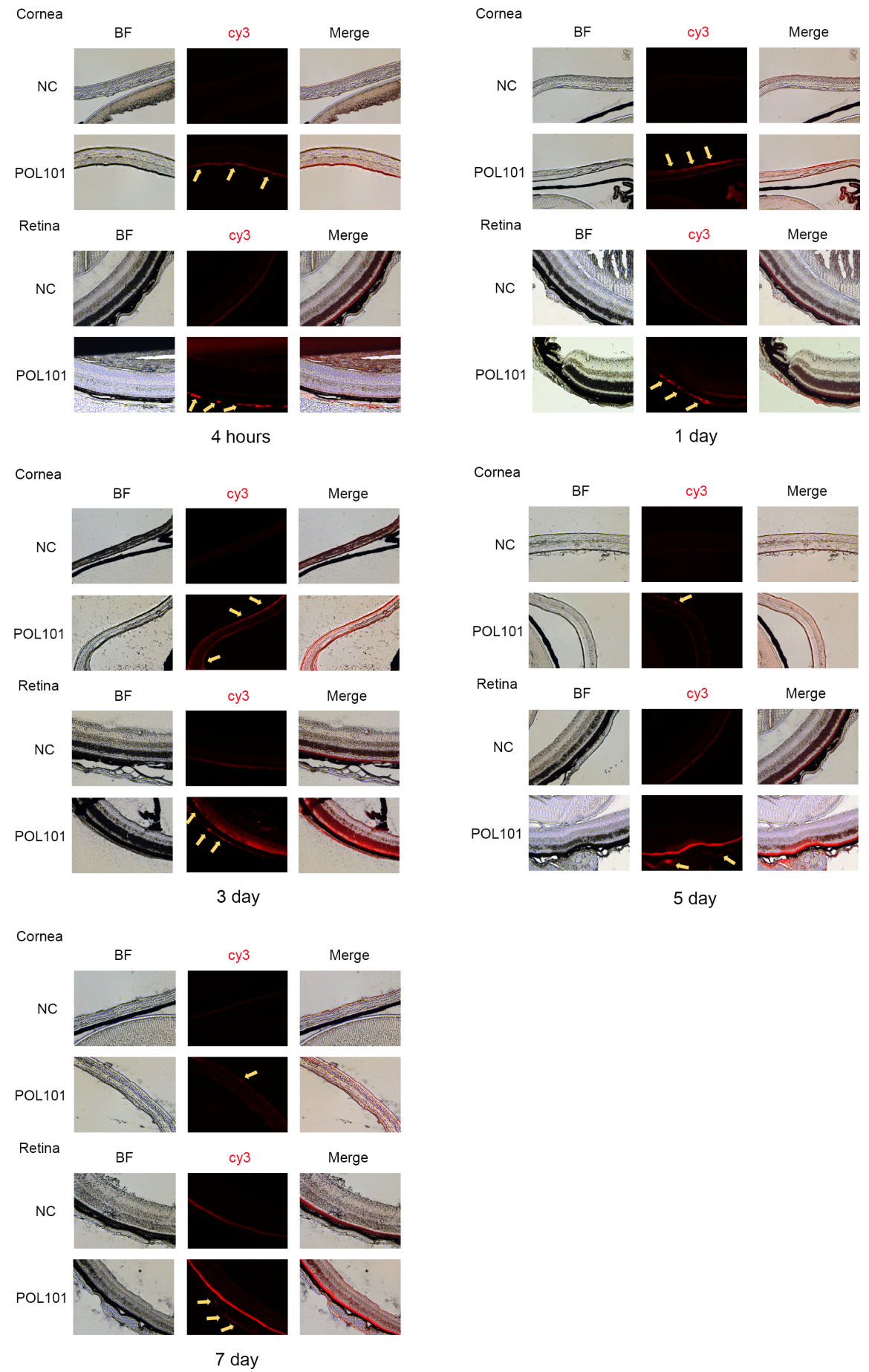
(a)

Vehicle
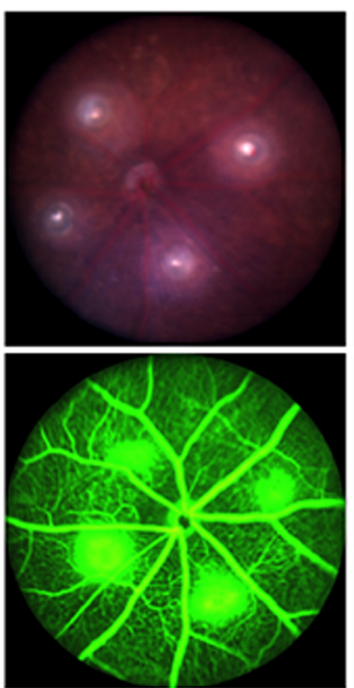

(b)

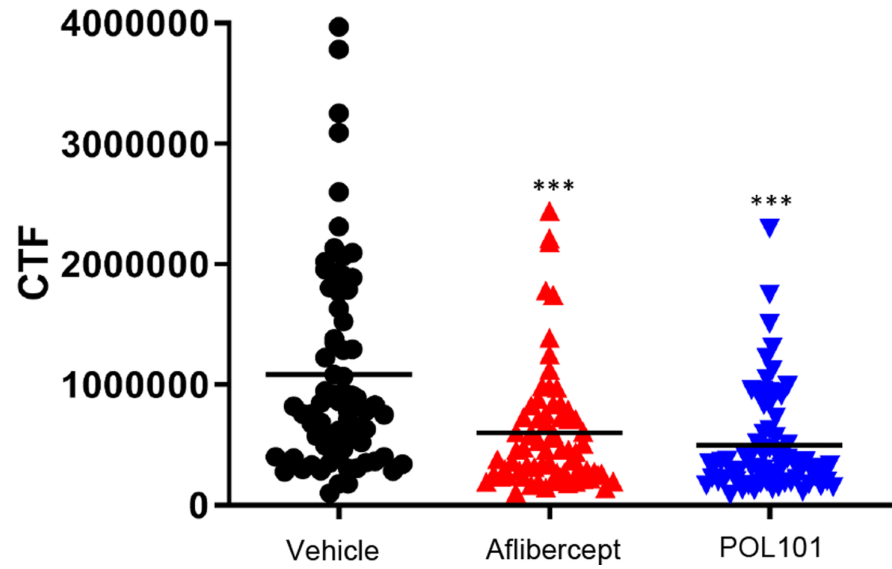


(a)

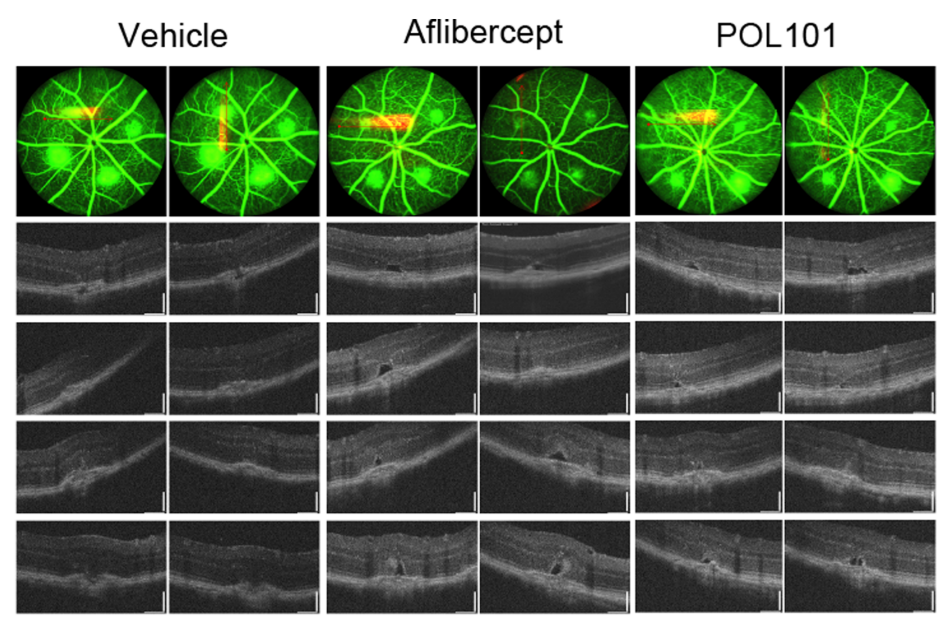

(b)

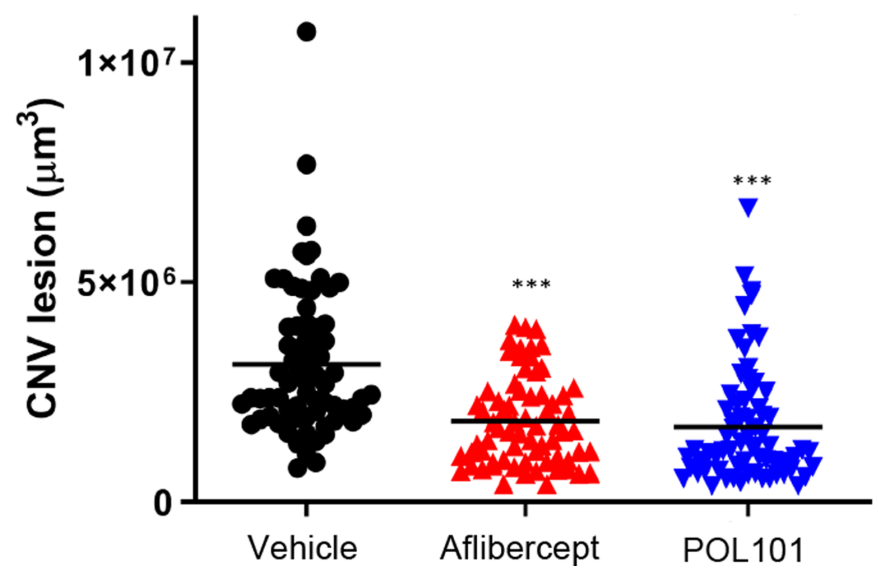


(a)

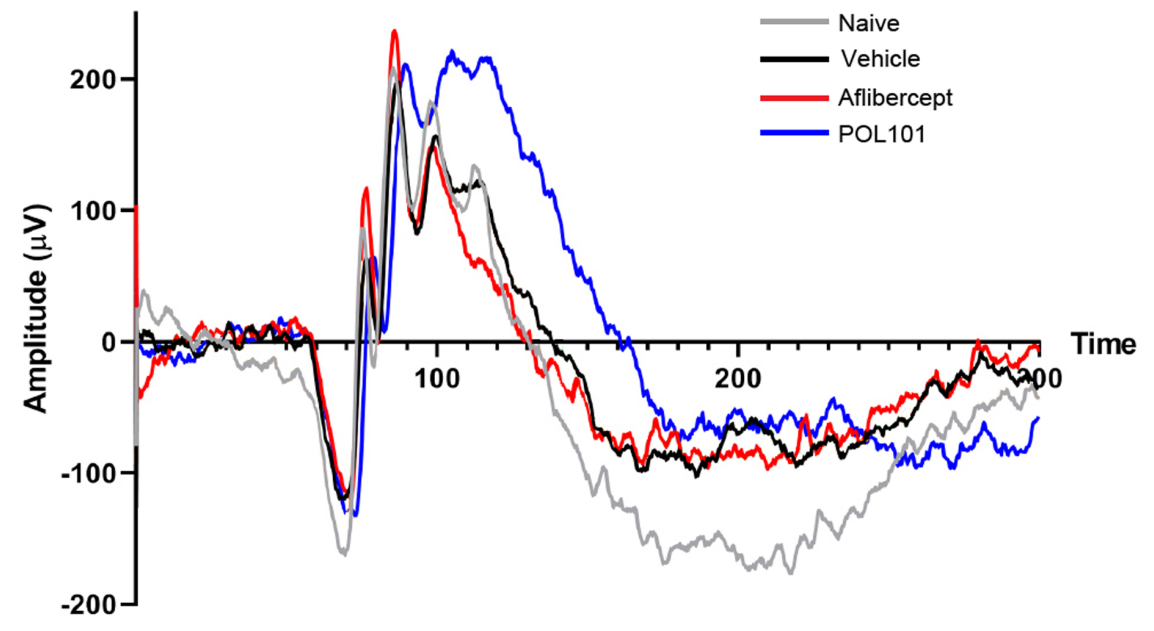

(b)

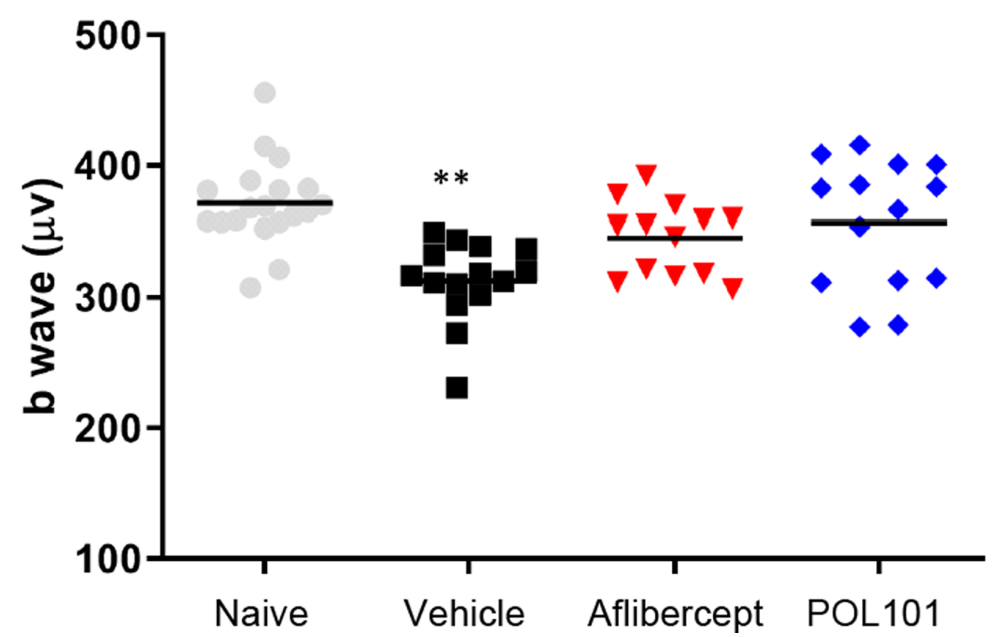


(a)

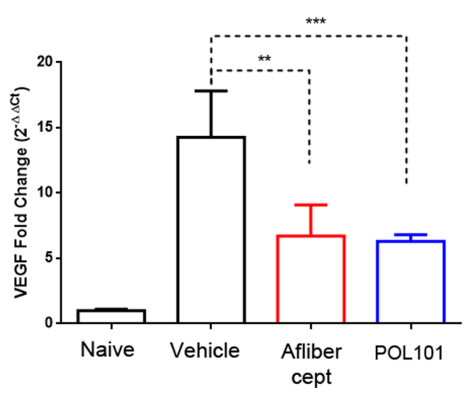

(c)

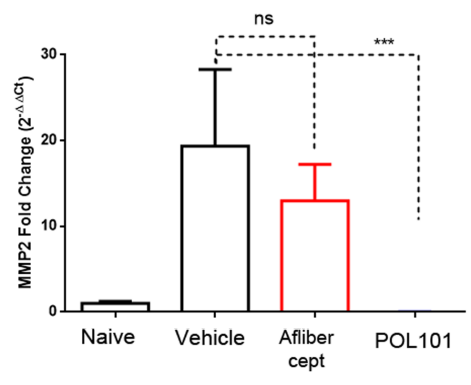

(b)

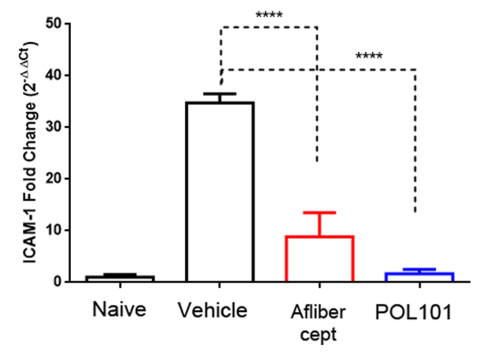

(d)

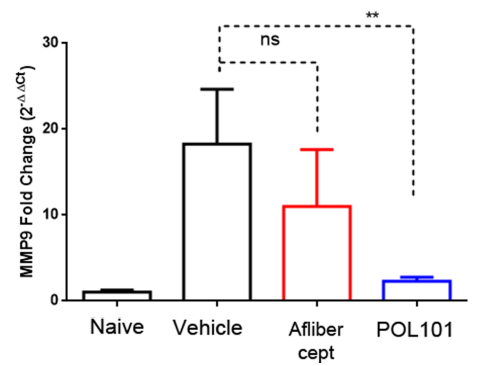


(a)

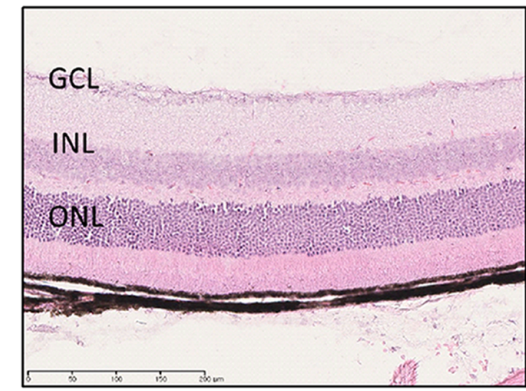

Naive

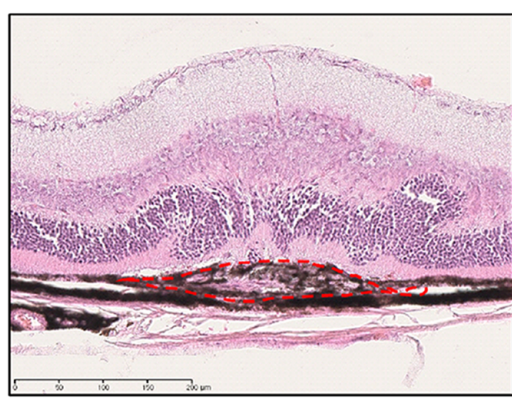

Aflibercept

(b)

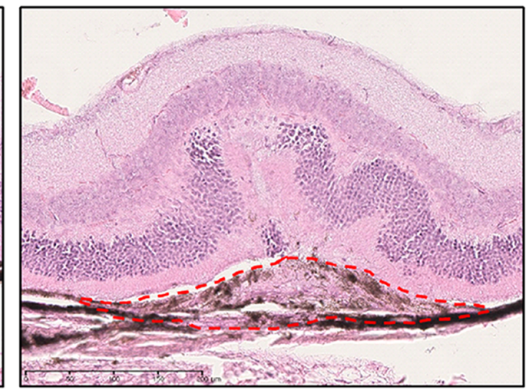

Vehicle

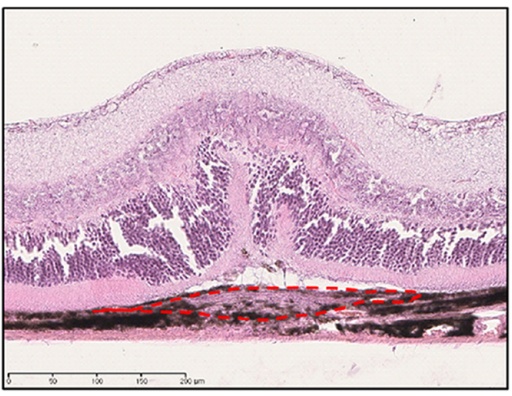

POL101

Scale bar : $200 \mu \mathrm{m}$

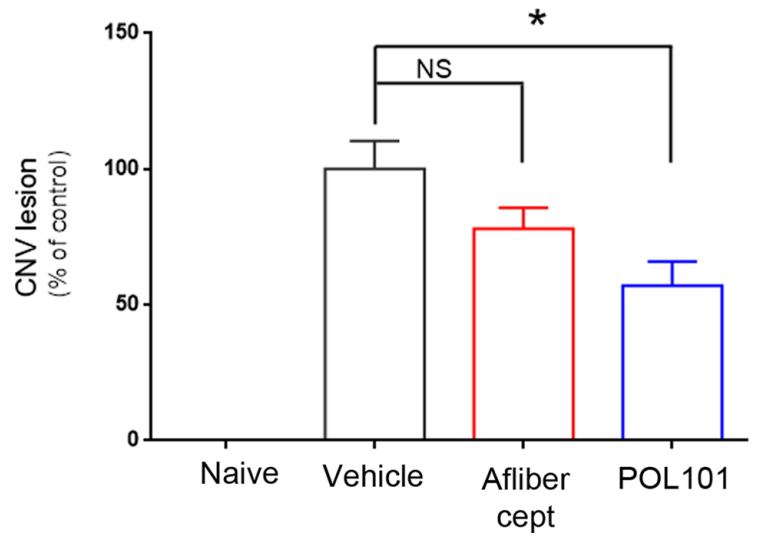


(a)

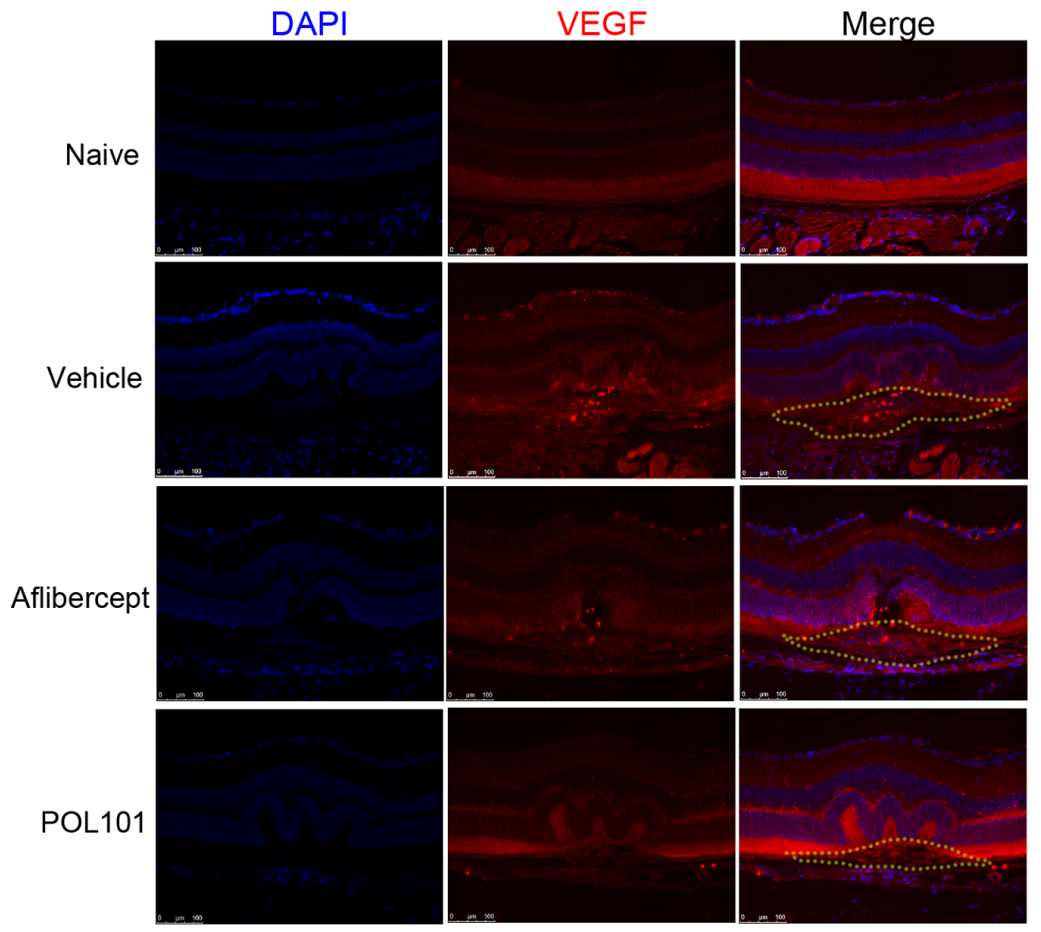

(b)

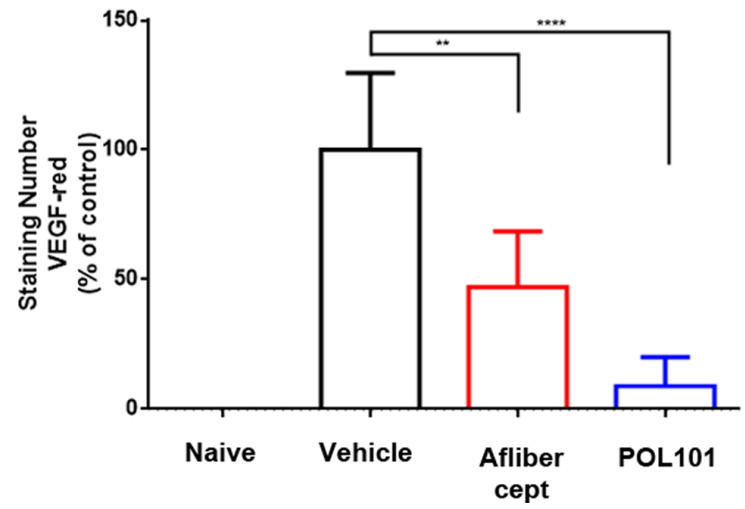


(a)

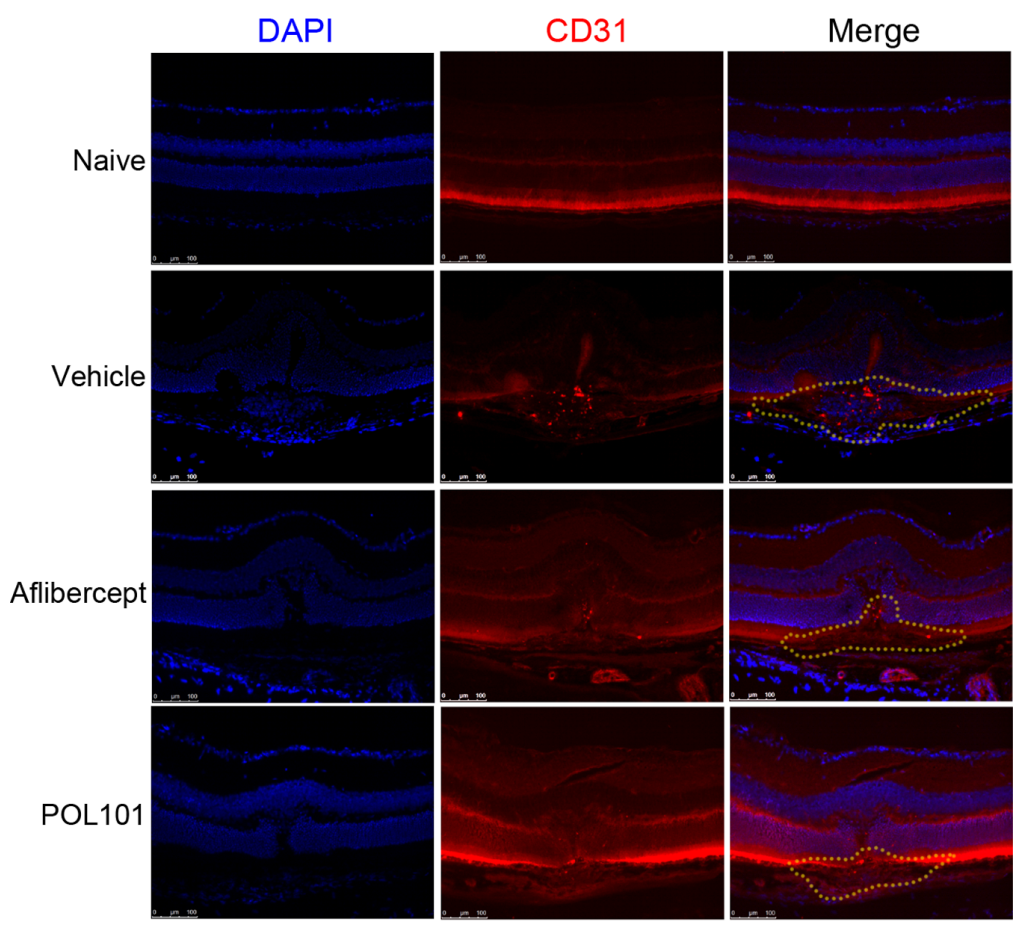

(b)

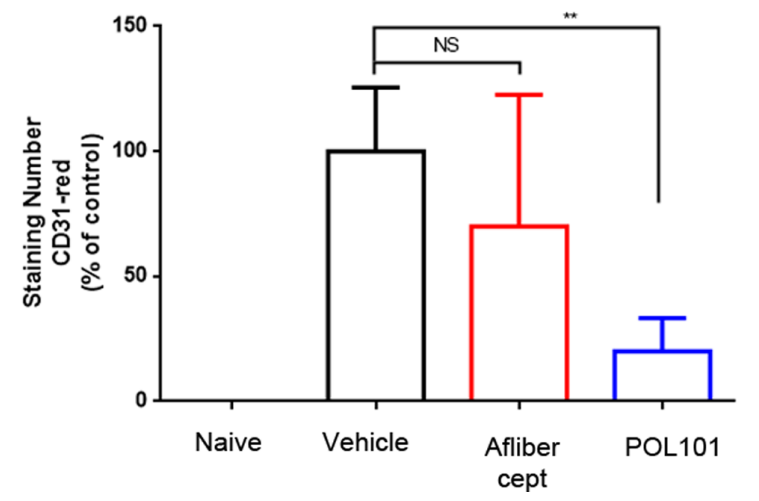

